\title{
PAPER \\ Evaluation of EMI Reduction Effect of Guard Traces Based on Imbalance Difference Model
}

\author{
Tohlu MATSUSHIMA ${ }^{\dagger a)}$, Student Member, Tetsushi WATANABE ${ }^{\dagger \dagger}$, Yoshitaka TOYOTA ${ }^{\dagger}$, Ryuji KOGA $^{\dagger}$, \\ and Osami WADA ${ }^{\dagger \dagger}$, Members
}

\begin{abstract}
SUMMARY Placing a guard trace next to a signal line is the conventional technique for reducing the common-mode radiation from a printed circuit board. In this paper, the suppression of common-mode radiation from printed circuit boards having guard traces is estimated and evaluated using the imbalance difference model, which was proposed by the authors. To reduce common-mode radiation further, a procedure for designing a transmission line with guard traces is proposed. Guard traces connected to a return plane through vias are placed near a signal line and they decrease a current division factor (CDF). The CDF represents the degree of imbalance of a transmission line, and a common-mode electromotive force depends on the CDF. Thus, by calculating the CDF, we can estimate the reduction in common-mode radiation. It is reduced not only by placing guard traces, but also by narrowing the signal line to compensate for the variation in characteristic impedance due to the guard traces. Experimental results showed that the maximum reduction in common-mode radiation was about $14 \mathrm{~dB}$ achieved by placing guard traces on both sides of the signal line, and the calculated reduction agreed with the measured one within $1 \mathrm{~dB}$. According to the $\mathrm{CDF}$ and characteristic impedance calculations, common-mode radiation can be reduced by about $25 \mathrm{~dB}$ while keeping the characteristic impedance constant by changing the gap between the signal line and the guard trace and by narrowing the width of the signal line.

key words: PCB, common-mode radiation, imbalance difference model, guard trace, optimum design
\end{abstract}

\section{Introduction}

Common-mode radiation dominates electromagnetic interference (EMI) from printed circuit boards (PCBs) [1]. A high-speed device on a PCB is intended to drive a functional signal. This signal, however, unintentionally produces significant electromagnetic interference through resonances determined by the shape of the structure. To suppress EMI below a prescribed level, a scheme for controlling EMI should be deployed at the PCB design stage.

A guard trace running near a signal line is used to reduce common-mode radiation from a PCB [2], [3]. The common-mode radiation increases when the return plane of the signal is narrow and/or the signal line runs close to the edges of the return plane [4], [5]. A guard trace that has the same potential as the return plane acts as additional a return-

Manuscript received August 4, 2008.

Manuscript revised December 5, 2008.

${ }^{\dagger}$ The authors are with the Graduate School of Natural Science and Technology, Okayama University, Okayama-shi, 7008530 Japan.

${ }^{\dagger}$ The author is with Industrial Technology Center of Okayama Prefecture, Okayama-shi, 701-1296 Japan.

${ }^{\dagger \dagger}$ The author is with the Graduate School of Engineering, Kyoto University, Kyoto-shi, 615-8510 Japan.

a)E-mail: matsushima@dev.cne.okayama-u.ac.jp

DOI: 10.1587/transcom.E92.B.2193

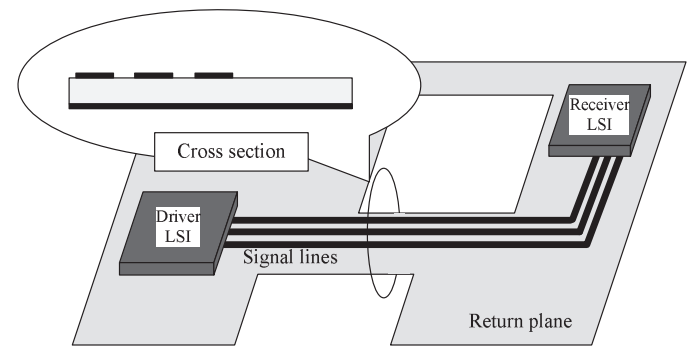

Fig. 1 A printed circuit board with a guard trace running near a signal line.

current path and can reduce the common-mode radiation.

The reduction in common-mode radiation obtained by using a guard trace can be evaluated directly through a fullwave electromagnetic simulation such as the FDTD method [6], which generally requires a lot of computing resources and time. To design PCBs with the guard traces, it is desirable to estimate the effect of the guard trace quantitatively and quickly. However, there has not been a fast calculation method for evaluating the reduction effect, which depends on the location and/or the number of guard traces.

The authors have proposed a mechanism for commonmode generation in which it is caused by a mismatch in a PCB's transmission line imbalance [7]-[9]. Based on this concept, the common-mode generation can be modeled in two phases: evaluation of the excitation source voltage and evaluation of the radiation from the common-mode antenna.

A guard trace can suppress the radiation from a line placed near the edge of a PCB, as shown in Fig. 1. The evaluation of the excitation source requires only a twodimensional calculation in our method, and we can repeatedly verify design parameters in a PCB layout design with guard traces to obtain a result that meets design requirements.

In this paper, the authors explain a method for calculating the reduction effect of guard traces and evaluate the method's prediction accuracy. In addition, we propose a procedure for designing transmission lines with guard traces to reduce common-mode radiation.

\section{Imbalance Difference Model}

The authors have proposed the imbalance difference model [8], [9] to predict of common-mode radiation from a PCB. In this model, the degree of imbalance of a transmission line 
is evaluated, and the variation in the imbalance affects the common-mode electromotive force.

In this section, we consider a microstrip transmission line, as shown in Fig. 2(a), to explain the imbalance difference model. This is the fundamental structure used to explain the mechanism for common-mode generation. This transmission line consists of two parts. In part a, the transmission line has a wide return plane. The cross-sectional structure between $\alpha$ and $\alpha^{\prime}$ is shown in Fig. 2(b). On the other hand, the return plane is narrow in part $b$, as shown in Fig. 2(c), which shows the cross-sectional structure of the transmission line between $\beta$ and $\beta^{\prime}$. The guard trace placed near the signal line is connected with the return plane. Therefore, the guard trace potential $V_{\mathrm{G}}$ is equal to the return plane potential $V_{\mathrm{R}}$. The microstrip structure on each part is thin enough that only the TEM mode propagates and the effects of higher-order modes are ignored.

\subsection{Current Division Factor and Common-Mode Potential}

To evaluate common-mode generation, we use a current division factor (CDF) [8], which represents the degree of imbalance of a transmission line. The CDF is the ratio of the common-mode current flowing on the signal line to the total common-mode current flowing on the transmission line. Therefore, the CDF is denoted as

$$
h=\frac{I_{\mathrm{CS}}}{I_{\mathrm{CS}}+I_{\mathrm{CR}}+I_{\mathrm{CG}}},
$$

where $I_{\mathrm{CS}}, I_{\mathrm{CR}}$ and $I_{\mathrm{CG}}$ are common-mode currents flowing on the signal line, return plane, and guard trace, respectively. When the transmission line consists of only the signal line and return plane, we consider that no current flows on the guard trace $\left(I_{\mathrm{CG}}=0\right)$. The CDF is determined from the cross-sectional structure of the transmission line. Therefore, the guard trace running near the signal line affects the imbalance of the transmission line. As far as the TEM mode is assumed, the CDF is independent of frequency.

When we focus on the only common-mode propagation, both the signal line and the return plane can be considered to be at the same potential $V_{C}$. The virtual commonmode potential $V_{\mathrm{C}}$ is derived by using the $\mathrm{CDF}$ [8] as

$$
V_{\mathrm{C}}=h V_{\mathrm{S}}+(1-h) V_{\mathrm{R}}=V_{\mathrm{R}}+h V_{\mathrm{N}},
$$

where $V_{\mathrm{S}}$ and $V_{\mathrm{R}}$ are signal line potential and return plane potential, respectively, and $V_{N}\left(=V_{S}-V_{R}\right)$ is the normalmode $^{\dagger}$ signal voltage.

Each part of the transmission line has a different cross section, so the CDF of each part has a different value. The common-mode potential in part a of the transmission line $V_{\mathrm{Ca}}$ is not equal to the common-mode potential in part $\mathrm{b}$, $V_{\mathrm{Cb}}$. At the interconnection of the two parts in the transmission line, as shown in Fig. 2(a), we assume an abrupt change in the common-mode potential, as shown in Fig. 2(d).

When the transmission lines are connected, there is a common-mode potential difference $\Delta V_{\mathrm{C}}$ at the interconnection,

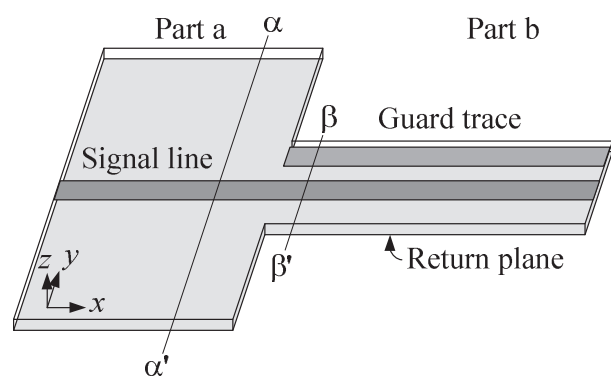

(a) Microstrip line with a guard trace.

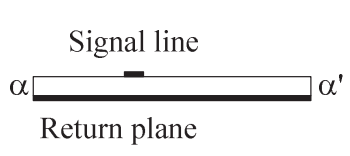

(b) Microstrip line with a large return plane.
Guard trace Signal line

$$
\beta \underset{\mathbf{T}-\square}{ } \beta^{\prime}
$$

(c) Microstrip line with a narrow return plane and a guard trace.

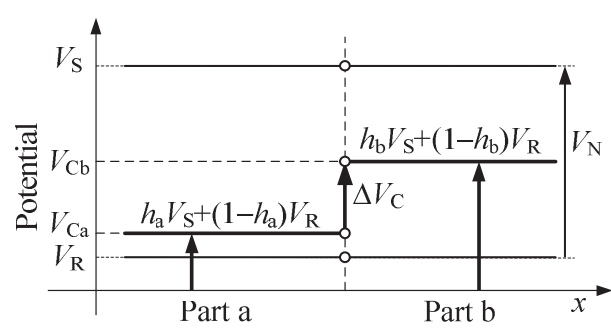

(d) Potential diagram near connection of transmission lines.

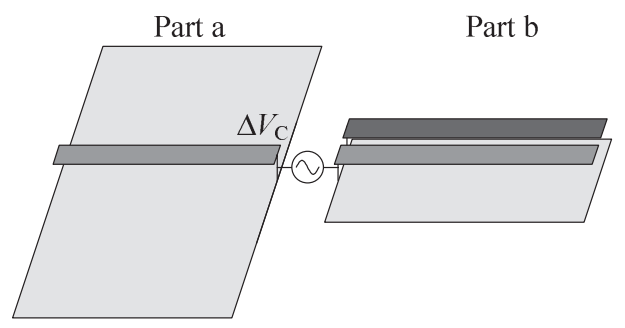

(e) Common-mode antenna.

Fig. 2 Imbalance difference model of microstrip line with a guard trace.

$$
\begin{aligned}
\Delta V_{\mathrm{C}} & =V_{\mathrm{Cb}}-V_{\mathrm{Ca}}, \\
& =\left(h_{\mathrm{b}}-h_{\mathrm{a}}\right)\left(V_{\mathrm{S}}-V_{\mathrm{R}}\right)=\Delta h V_{\mathrm{N}},
\end{aligned}
$$

where $h_{\mathrm{a}}$ and $h_{\mathrm{b}}$ are the CDFs in the part a and the part $\mathrm{b}$, respectively, and $\Delta h$ is the difference in the CDF. Thus, the two parts, part a and part $b$, are excited by the commonmode potential difference $\Delta V_{\mathrm{C}}$ in between, and commonmode current flows [8], [9].

\footnotetext{
${ }^{\dagger}$ Normal mode is explained in Appendix.
} 


\subsection{Common-Mode Radiation}

Common-mode current is excited by the common-mode potential difference $\Delta V_{\mathrm{C}}$ in a common-mode equivalent circuit and the current causes common-mode radiation. When a PCB is located far from other metal objects, the PCB acts as an antenna. This antenna, which we call the "common-mode antenna," is shown in Fig. 2(e). For the normal mode, the dielectric between the trace and the return plane has a large effect because most of the electromagnetic field is kept in the dielectric. However, the effect of the dielectric is small for the common mode, and we can ignore the dielectric. Moreover, most of the common-mode current usually flows on the return plane due to the low impedance of the plane. Therefore, the shape of the return plane and the location of the excitation sources are the dominant factors in common-mode radiation from test boards, and we ignore the signal line and the guard traces to obtain a simplified common-mode antenna.

The common-mode radiation $\boldsymbol{E}(\boldsymbol{r}, f)$ from the commonmode antenna can be calculated by the following equation [8],

$$
\begin{aligned}
\boldsymbol{E}(\boldsymbol{r}, f) & =\Delta V_{\mathrm{C}} \cdot \boldsymbol{F}(\boldsymbol{r}, f), \\
& =\Delta h \cdot V_{\mathrm{N}} \cdot \boldsymbol{F}(\boldsymbol{r}, f),
\end{aligned}
$$

where $\boldsymbol{F}(\boldsymbol{r}, f)$ is an antenna radiation factor. This factor is obtained by numerical analysis and is determined by the structure of the antenna when the antenna is excited by a unit voltage source. Here, $\boldsymbol{r}$ and $f$ are the position vector of the observation point and the frequency, respectively.

According to Eq. (6), the radiated emission is the product of the imbalance difference $\Delta h$, the normal-mode voltage $V_{\mathrm{N}}$, and the antenna radiation factor $\boldsymbol{F}(\boldsymbol{r}, f)$. Strictly speaking the antenna radiation factors $\boldsymbol{F}(\boldsymbol{r}, f)$ of the test boards with or without the guard trace are different because the antenna structures differ from one another. As far as the CDF is small enough, however, most of the common-mode current flows on the return plane and hence the common-mode antenna structure is dominantly determined by the shape of the return plane. With this assumption, the antenna factor $\boldsymbol{F}(\boldsymbol{r}, f)$ can be considered as still the same regardless of the guard trace as long as the shape of the return plane is the same.

If the normal-mode voltage and the antenna factor are kept unchanged, the common-mode radiation is proportional to only the difference in the CDF, $\Delta h$. Therefore, the difference in the common-mode radiations $\Delta \boldsymbol{E}$ from the transmission line with and without the guard trace is given by the difference in $\Delta h$. Therefore we estimate the reduction of common-mode radiation by calculating the difference in $\Delta h$.

\subsection{CDF Calculation Method}

If we only consider the common mode propagating along

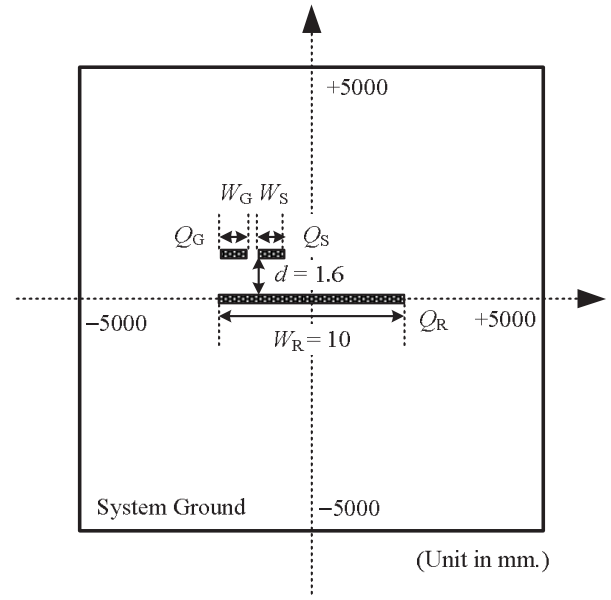

Fig. 3 Analysis model for CDF of a transmission line.

the transmission line as a TEM mode, the CDF in Eq. (1) is expressed by the transmission line parameters per unit length [8]. When the guard trace has the same potential as the return plane, the CDF is written as

$$
h=\frac{C_{\mathrm{SS}}}{C_{\mathrm{SS}}+C_{\mathrm{RR}}+C_{\mathrm{GG}}},
$$

where $C_{\mathrm{SS}}, C_{\mathrm{RR}}$, and $C_{\mathrm{GG}}$ are self capacitances[10] of the signal line, the return plane and the guard trace to the system ground, respectively.

Also, the CDF can be rewritten as

$$
h=\frac{Q_{\mathrm{S}}}{Q_{\mathrm{S}}+Q_{\mathrm{R}}+Q_{\mathrm{G}}},
$$

where $Q_{\mathrm{S}}, Q_{\mathrm{R}}$, and $Q_{\mathrm{G}}$ are the charges per unit length on the signal line, the return plane and, the guard trace, respectively, calculated through two-dimensional static field analysis when potentials of three conductors are set to a unit voltage, as shown in Fig. 3. In the transmission line without a guard trace, the charge on the guard trace $Q_{\mathrm{G}}$ in Eq. (8) is set to 0 .

The CDF $h$ decreases when a transmission line has the wide return plane because $C_{\mathrm{RR}}$ increases. In addition, when a guard trace is placed along the signal line, $C_{\mathrm{GG}}$ increase, and $C_{\mathrm{RR}}$ decreases as well as $C_{\mathrm{SS}}$. Accordingly, the denominator in Eq. (7) increases and the numerator decreases, and the CDF decreases when the guard trace is placed.

\section{Prediction of Common-Mode Radiation}

In this section, the reduction in common-mode radiation obtained by using guard traces is evaluated from CDF calculations and from measurements of the emissions radiated from test boards.

\subsection{Test Boards}

The reduction in the common-mode radiation was examined using the test board depicted in Fig. 4. In actual PCBs, transmission lines have several discontinuities, such as a narrow 


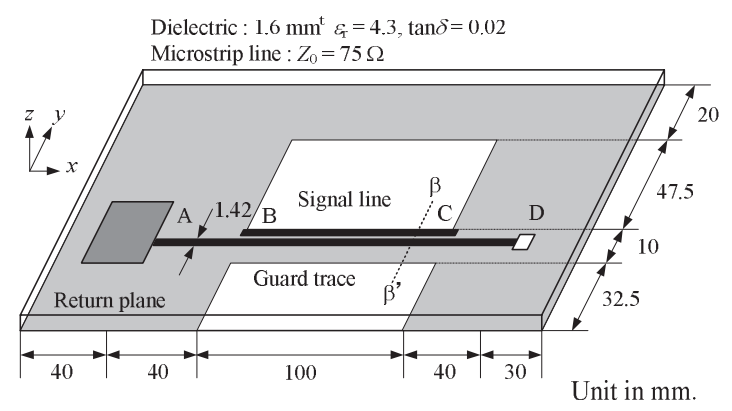

Fig. 4 Structure of a test board with a guard trace on inside.

return plane for high speed signal or a signal line running near the edge of a return plane. The test board simulates actual PCBs a the narrow return plane. The width of the return plane in the test board changes at two points, B and C.

To compare reduction effects of the guard trace, six types of test boards were used in the measurement. Each of the boards consisted of two layers: the top layer for the signal line and the bottom layer for the return plane. A straight signal line was placed along the $x$ direction between $\mathrm{A}$ and $\mathrm{D}$ for simplicity; between $\mathrm{B}$ and $\mathrm{C}$, the signal line ran above the narrow return plane. The cross-sectional structure of the transmission line changed at the locations $\mathrm{B}$ and $\mathrm{C}$. Therefore, the CDFs of the transmission line changed abruptly and common-mode excitation sources were generated at these points. At point A, the microstrip line was driven by a driver module with a CMOS IC (74AC00) and a 10-MHz crystal oscillator, supplied by a battery module placed on the bottom-layer side. The signal line was terminated with a matched load at point D.

The reduction in common-mode radiation was evaluated from the difference in radiation from test boards with and without guard traces. Six types of test boards, as shown in Fig. 5 were examined:

(a) without guard trace (reference)

(b) with an inside guard trace

(c) with an outside guard trace

(c') with an outside guard trace with a narrow signal line $\left(W_{\mathrm{S}}=0.7 \mathrm{~mm}\right)$

(d) with guard traces on both sides

(d') with guard traces on both sides with a narrow signal line $\left(W_{\mathrm{S}}=0.4 \mathrm{~mm}\right)$

The cross-sectional structures $\left(\beta-\beta^{\prime}\right.$ in Fig. 4$)$ between $\mathrm{B}$ and $\mathrm{C}$ were distinct from each other. The guard traces ran along the signal line between $\mathrm{B}$ and $\mathrm{C}$ in the test boards except for test board (a) and were connected to the return plane through the via holes at locations $\mathrm{B}$ and $\mathrm{C}$.

The widths of the signal line in sections A-B and C$\mathrm{D}$ were both $1.4 \mathrm{~mm}$. The gap between the signal line and the guard trace was $0.3 \mathrm{~mm}$ and the other dimensions of the transmission line structure are listed in Table 1. The characteristic impedance of the microstrip line on the reference test board was approximately $75 \Omega$.

Here, we discuss the interval of vias. Since the guard

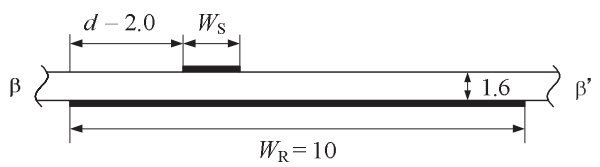

(a) reference board.

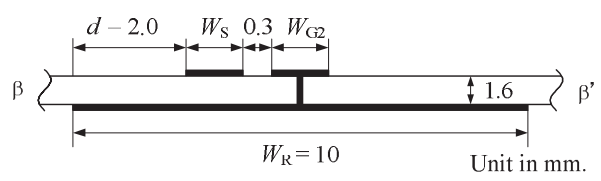

(b) with a guard trace on inside.

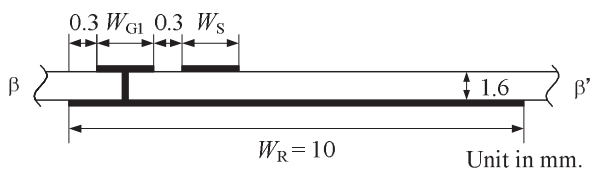

(c) with a guard trace on outside.

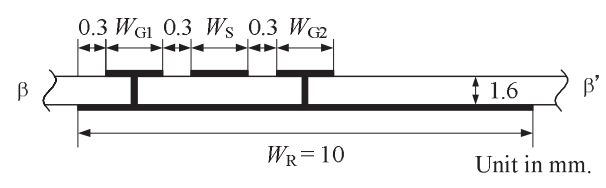

(d) with guard traces on both sides.

Fig. 5 Cross-sectional structures of test boards.

Table 1 The width of the signal line and the guard trace(s) and characteristic impedances in test boards.

\begin{tabular}{ccccc}
\hline Type & $\begin{array}{c}W_{\mathrm{G} 1} \\
{[\mathrm{~mm}]}\end{array}$ & $\begin{array}{c}W_{\mathrm{S}} \\
{[\mathrm{mm}]}\end{array}$ & $\begin{array}{c}W_{\mathrm{G} 2} \\
{[\mathrm{~mm}]}\end{array}$ & $\begin{array}{c}Z_{0} \\
{[\Omega]}\end{array}$ \\
\hline \hline (a) & - & 1.4 & - & 75 \\
\hline (b) & - & 1.4 & 1.4 & 59 \\
\hline (c) & 1.4 & 1.4 & - & 59 \\
\hline$\left(\mathrm{c}^{\prime}\right)$ & 1.4 & 0.7 & - & 75 \\
\hline$(\mathrm{d})$ & 1.4 & 1.4 & 1.4 & 50 \\
\hline$\left(\mathrm{d}^{\prime}\right)$ & 1.4 & 0.4 & 1.4 & 74 \\
\hline
\end{tabular}

trace is grounded at locations B and C, the half-wavelength resonance is the first resonance of the guard trace. Hence, the guard trace stays at the same potential as the return plane as far as the interval of vias is shorter than the half wavelength. The frequency where the interval of vias is the half wavelength is calculated as

$$
f_{\frac{\lambda}{2}}=\frac{c_{0}}{2 \ell \sqrt{\varepsilon_{\mathrm{eff}}}}
$$

where $c_{0}, \ell$ and $\varepsilon_{\text {eff }}$ are the velocity of light in vacuum, the interval of vias and effective relative permittivity, respectively. In the test boards, the half wavelength resonance frequency of the guard trace is about $800 \mathrm{MHz}$ because the relative permittivity of the test boards is 4.3 (FR-4). Therefore, we consider the guard trace keeps the same potential to the return plane below $800 \mathrm{MHz}$ since the guard trace is 
Table 2 CDFs of the test boards and estimation of common-moderadiation reduction.

\begin{tabular}{cccc}
\hline Type & $h_{\mathrm{BC}}$ & $\begin{array}{c}\Delta E[\mathrm{~dB}] \\
\text { (Calc. })\end{array}$ & $\begin{array}{c}\Delta E_{\text {avg }}[\mathrm{dB}] \\
\text { (Meas. })\end{array}$ \\
\hline \hline (a) & 0.151 & Reference & Reference \\
\hline (b) & 0.116 & -2.3 & -3.2 \\
\hline (c) & 0.095 & -4.0 & -4.3 \\
\hline (c $\left.{ }^{\prime}\right)$ & 0.067 & -7.1 & -6.5 \\
\hline (d) & 0.065 & -7.3 & -7.6 \\
\hline$\left(\mathrm{d}^{\prime}\right)$ & 0.028 & -14.6 & -13.7 \\
\hline
\end{tabular}

grounded at locations $\mathrm{B}$ and $\mathrm{C}$.

\subsection{Estimation of Common-Mode Radiation}

In the reference test board, shown in Fig. 5(a), the CDF of the transmission line with the narrow return plane was 0.151 which is higher than that of the transmission line having a wide return plane in $\mathrm{A}-\mathrm{B}$ and $\mathrm{C}-\mathrm{D}$. Reduction of commonmode radiation was estimated by calculating $\mathrm{CDFs}$, which are shown in Table 2.

The CDF of the transmission line with the wide return plane was approximately equal to 0 . Therefore, the imbalance difference $\Delta h$ at the locations $\mathrm{B}$ and $\mathrm{C}$ has the same value as the $\mathrm{CDF}$ of the transmission line between $\mathrm{B}$ and $\mathrm{C}$, $h_{\mathrm{BC}}$. When the guard trace was placed near the signal line between $\mathrm{B}$ and $\mathrm{C}$, as shown in Fig. 5(b), $\Delta h$ at interconnections $\mathrm{B}$ and $\mathrm{C}$ was 0.116 . Since the difference in commonmode radiation from test boards (a) and (b) was proportional to $\Delta h$, the reduction in common-mode radiation was calculated as $0.116 / 0.151=-2.3 \mathrm{~dB}$.

Test board (c), which is shown in Fig. 5(c), had a guard trace near the edge of the return plane. The CDF $h_{\mathrm{BC}}$ was reduced to 0.095 . In this case, the difference in common-mode radiation from the test board (a) was reduced to $0.095 / 0.151$ $=-4.0 \mathrm{~dB}$, which was lower than that for the test board (b). Test board (d), which is shown in Fig. 5(d), reduced the common-mode radiation more than the radiation from test board (b) or (c) because there guard traces were placed on the both sides (one on each side) of the signal line. The CDF $h_{\mathrm{BC}}$ of test board (d) was 0.065 , and the difference from test board (a) was $0.065 / 0.151=-7.3 \mathrm{~dB}$.

The narrow signal line achieved a larger reduction in common-mode radiation. Test boards $\left(c^{\prime}\right)$ and $\left(d^{\prime}\right)$ had a narrow signal line between $B$ and $C$. The signal line widths of test board $\left(\mathrm{c}^{\prime}\right)$ and $\left(\mathrm{d}^{\prime}\right)$ were $0.7 \mathrm{~mm}$ and $0.4 \mathrm{~mm}$, respectively, as listed in Table 1. The characteristic impedances of test boards (c) and (d) were decreased to 59 and $50 \Omega$, respectively, by the guard trace running near the signal line. The width of the signal line was then narrowed for impedance matching, and the CDF of the transmission line between $\mathrm{B}$ and $\mathrm{C}$ decreased as the same time. The CDF $h_{\mathrm{BC}}$ of test board $\left(\mathrm{d}^{\prime}\right)$ having two guard traces and a narrow signal line was 0.028 , and the difference in radiation relative to test board (a) was $0.028 / 0.151=-14.6 \mathrm{~dB}$.

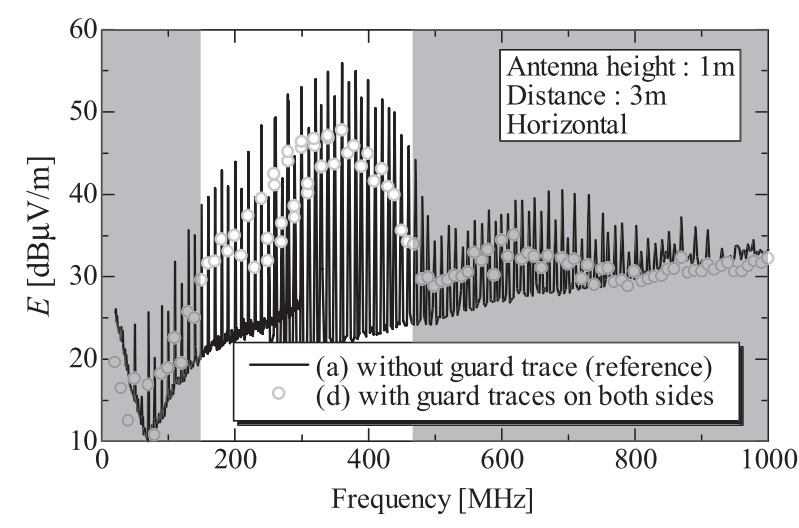

(a) Common-mode radiation from test boards.

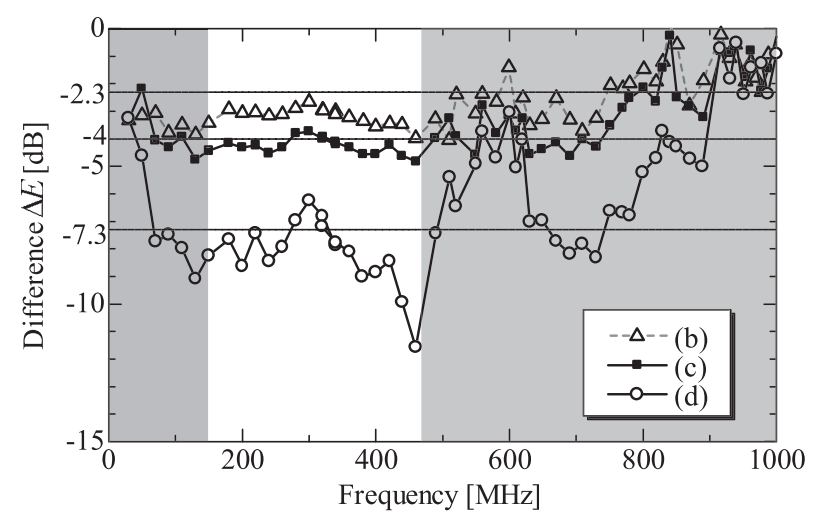

(b) Common-mode radiation reduction by using guard trace.

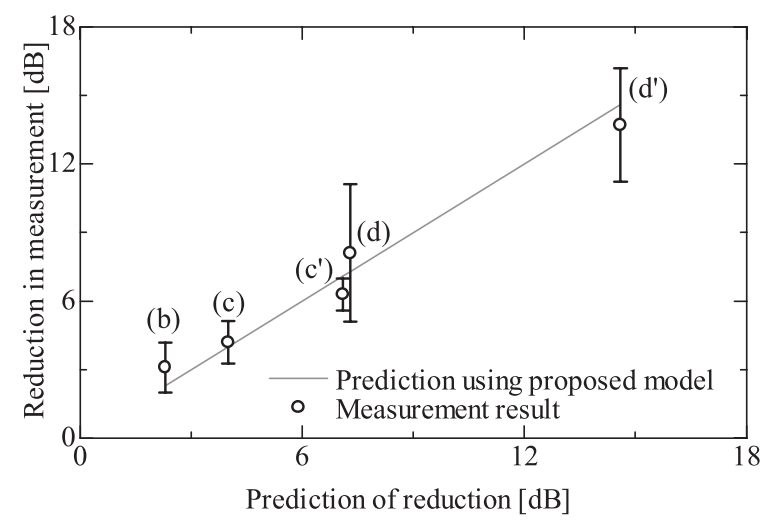

(c) Estimation of difference between radiations from reference board and other boards.

Fig. 6 Reduction of common-mode radiation from test boards using guard trace.

3.3 Verification of Prediction of Common-Mode Radiation

Common-mode radiation from two test boards, (a) and (d), measured at a distance of $3 \mathrm{~m}$ in a semi-anechoic chamber 
is shown in Fig. 6(a). The test boards were positioned with the $x y$-plane parallel to the floor on a rectangular block of polystyrene foam $1 \mathrm{~m}$ in height. The receiving antenna was located at a point $3 \mathrm{~m}$ from the test board in the $-y$ direction, and the antenna height was fixed at $1 \mathrm{~m}$ above the chamber floor during this measurement.

Figure 6(b) shows the variations of the radiated electric field strength $\Delta E$ with the guard trace, obtained by comparing with the field from the reference board. The values -2.3 , -4 , and -7.3 dB in Fig. 6(b) are the reduction ratio obtained by the CDFs calculation. We are interested in the frequency range from 140 to $470 \mathrm{MHz}$ because the field strength of radiation from test board (d) in other frequency bands (shaded areas in Fig. 6) was less than $10 \mathrm{~dB}$ above the noise floor in the measurement and has low accuracy of measurement for evaluation of the reduction of the common-mode radiation. The reductions in common-mode radiation for test boards are shown in Table 2. Each of the $\Delta E_{\text {avg }}$ (Meas.) values is the geometric average of the reductions in common-mode radiation in the frequency band from 140 to $470 \mathrm{MHz}$. In addition, we estimate the measurement results of the reduction as shown in Fig. 6(c). In this Figure, a solid line shows reduction calculated by $\mathrm{CDF}$, and black circles plot measurement results. Each bar has a range of $\pm 2 \sigma$ where $\sigma$ is standard deviation. The measurement results have good agreement with the calculation results.

As a result, the reduction in common-mode radiation achieved by using the guard trace is predicted within $1 \mathrm{~dB}$ and is achieved within a few seconds by calculating the CDFs. Therefore, the imbalance difference model can be used for optimizing the design of the guard traces to reduce the common-mode radiation.

\section{Optimized Design of Guard Traces to Reduce Common-Mode Radiation}

The characteristic impedance and the CDF of the transmission line, which are shown in Fig. 7, in the case of two guard traces running on both sides of the signal line depend on the width of the signal line. Symbols (a), (d), and ( $\left.\mathrm{d}^{\prime}\right)$ in Fig. 7 show the types of test boards discussed in the previous section. When the guard trace is placed along the signal line and positions close to the signal line, the characteristic impedance decreases due to an increase in the capacitance between the signal line and the return plane together with the guard trace. Here, the guard trace acted as part of the return path. At the same time, the CDF is decreased by the placement of the guard trace.

To increase the characteristic impedance, the width of the signal line should be narrowed. Furthermore, the CDF decreases more because less electrical charge accumulates on the conductor of the signal line. The reasons the characteristic impedance increase as the width of the signal line becomes narrower are that the effective inductance of the signal line increases and the capacitance between the signal line and the return plane decreases. The difference between test boards (d) and ( $\left.\mathrm{d}^{\prime}\right)$ shows that the narrowing the signal

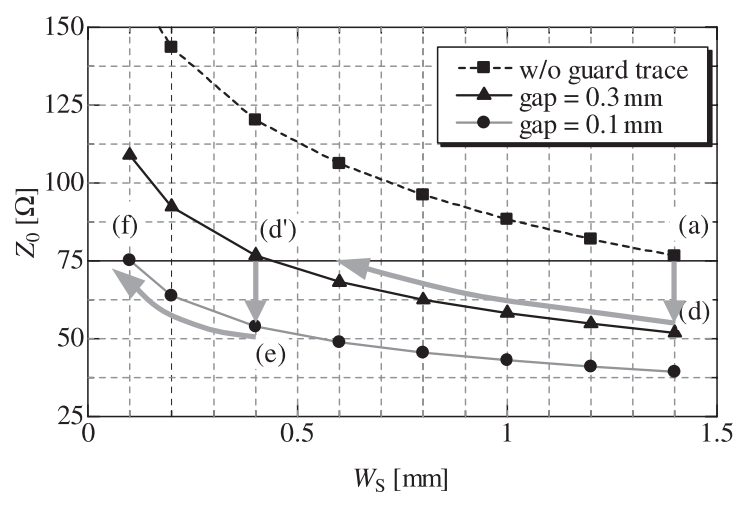

(a) Characteristic impedances calculated by using finite element method.

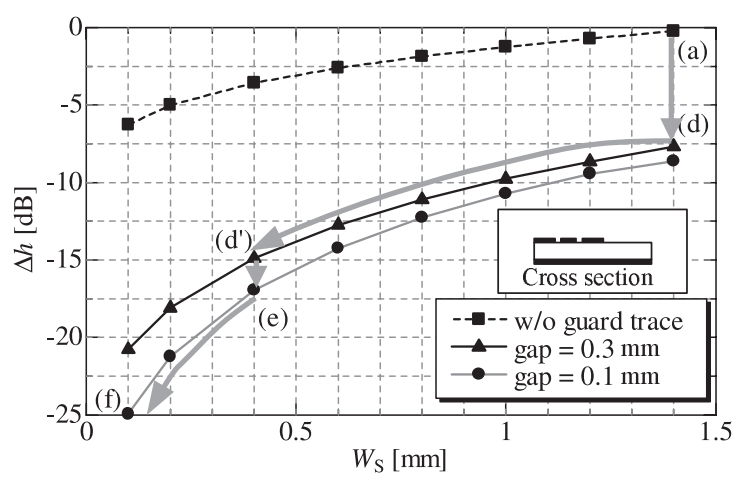

(b) Difference of CDFs, which were calculated by Eq. (8), between reference and transmission line with guard trace.

Fig. 7 Characteristic impedances and CDFs of the transmission line with guard traces running both sides of the signal line when width of the signal line changes.

line can decrease the CDF while keeping the characteristic impedance of the transmission line at $75 \Omega$.

A narrower gap between the signal line and the guard trace decreases the CDF and the characteristic impedance as shown in Fig. 7. For example, the CDF of test board ( $\left.\mathrm{d}^{\prime}\right)$ is decreased by about $2 \mathrm{~dB}$ when the gap is narrowed from $0.3 \mathrm{~mm}$ to $0.1 \mathrm{~mm}$ (from $\left(\mathrm{d}^{\prime}\right)$ to (e) in Fig. 7). At the same time, the characteristic impedance is decreased to approximately $55 \Omega$. The narrower signal line for keeping the characteristic impedance of $75 \Omega$ decreases the CDF. The radiation from test board with a $0.1-\mathrm{mm}$-wide signal line (symbol (f) in Fig. 7) is reduced by $25 \mathrm{~dB}$ from that of reference test board (a).

As a result, both placing the guard trace and narrowing signal line contribute to common-mode radiation reduction due to the decreases in the CDF of the transmission line. As for the characteristic impedance, on the other hand, placing the guard trace decreases it and narrowing the signal line increases it. Therefore, placing the guard trace with narrowing the signal line can realize reduction of common-mode radiation and keeping the characteristic impedance at $75 \Omega$ at the same time. 
The reduction in common-mode radiation varies depending on the cross-sectional structure of the transmission line, and it can be calculated quickly by using the imbalance difference model.

\section{Conclusion}

In this paper, the mechanism of the reduction in commonmode radiation achieved using guard traces was explained using the imbalance difference model. We discussed the estimation and evaluation of common-mode radiation reduction. Moreover, the authors proposed a procedure for designing a transmission lines with guard traces to reduce common-mode radiation even more.

The mechanism of common-mode radiation due to imperfection in the return plane can be explained in terms of the inconsistency of the transmission line imbalance. The common-mode radiation is caused by three factors: normalmode voltage, difference of imbalance, radiation factor of the common-mode current; these can be calculated separately. The imbalance difference can be controlled by guard traces placed adjacent to the signal line. Therefore, the guard traces reduce the imbalance difference and the common-mode radiation. Experiments found that the emission radiated from a test board with guard traces was reduced by about $14 \mathrm{~dB}$ by the addition of guard traces. The effect of the guard trace can be predicted to within an error of $1 \mathrm{~dB}$ by calculating the imbalance.

The effect of the guard traces can be estimated quantitatively and quickly by calculation of the CDFs. Our calculations showed that a $25-\mathrm{dB}$ reduction in common-mode radiation can be obtained by using guard traces while keeping the characteristic impedance of $75 \Omega$.

\section{References}

[1] D.M. Hockanson, J.L. Drewniak, T.H. Hubing, T.P. Van Doren, F. Sha, and M.J. Wilhelm, "Investigation of fundamental EMI source mechanisms driving common-mode radiation from printed circuit boards with attached cables," IEEE Trans. Electromagn. Compat. vol.38, no.4, pp.557-566, Nov. 1996.

[2] D.S. Britt, D.M. Hockanson, F. Sha, J.L. Drewniak, T.H. Hubing, and T.P. Van Doren, "Effects of gapped groundplanes and guard traces on radiated EMI," Proc. IEEE Int. Symp. Electromagnetic Compatibility, pp.159-164, Austin, TX, Aug. 1997.

[3] F.B. Leferink, "Reduciton of printed circuit board radiated emission," Proc. IEEE Int. Symp. Electromagnetic Compatibility, pp.431-438, Austin, TX, Aug. 1997.

[4] I.F. Chen and C.W. Hsue, "Evaluation of common-mode radiation from printed circuit boards by modelling imperfect ground effect," IEICE Trans. Commun., vol.E85-B, no.12, pp.2924-2933, Dec. 2002.

[5] K. Sasabe, A. Bullivant, K. Yoshida, and O. Fujiwara, "Prediction of electric far-field strength from printed circuit boards by measuring the common-mode current," Proc. IEEE Int. Symp. Electromagnetic Compatibility, pp.379-384, Washington, DC, Aug. 2000.

[6] Y. Kayano, M. Tanaka, and H. Inoue, "PCB structure with a guard band for suppressing electromagnetic radiation," IEICE Trans. Commun., vol.E88-B, no.8, pp.3182-3188, Aug. 2005.

[7] T. Watanabe, O. Wada, A. Namba, K. Fujimori, S. Matsunaga, and R. Koga, "Quantitative evaluation of common-mode radiation from a PCB based on imbalance difference model," Proc. Int. Symp. Electromagnetic Compatibility, pp.201-204, Sendai, Japan, June 2004.

[8] T. Watanabe, H. Fujihara, O. Wada, R. Koga, and Y. Kami, "A prediction method of common-mode excitation on a printed circuit board having a signal trace near the ground edge," IEICE Trans. Commun., vol.E87-B, no.8, pp.2327-2334, Aug. 2004.

[9] T. Watanabe, O. Wada, T. Miyashita, and R. Koga, "Common-modecurrent generation caused by difference of unbalance of transmission lines on a printed circuit board with narrow ground pattern," IEICE Trans. Commun., vol.E83-B, no.3, pp.593-599, March 2000.

[10] C.R. Paul, Analysis of Multiconductor Transmission Lines, ch. 3 , pp.69-73, John Wiley \& Sons, New York, 1994

\section{Appendix: Difference in Meaning between Normal Mode and Differential Mode}

For a symmetric balanced transmission line, as shown in Fig. A.1(a), a differential voltage generates a differential current. However, when the transmission line system is not completely balanced, as shown in Fig. A. 1(b), the voltage differential source does not always generate a differential current, and some non-differential current flows. To distinguish the current differential mode on an unbalanced transmission line from the differential mode on a balanced transmission line, we call the current differential mode on a general transmission line as the "normal mode", which is shown in Fig. A. 1(b). (a) Structure of symmetric balanced transmission line.

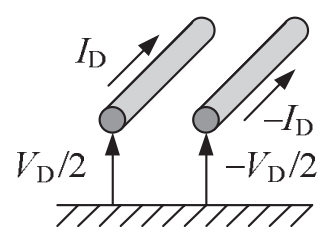

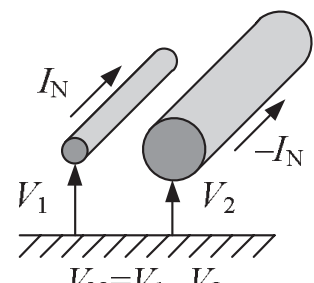

$V_{\mathrm{N}}=V_{1}-V_{2}$

(b) Structure of asymmetric transmission line.
Fig. A- 1 Differential-mode and normal-mode voltage and current on the transmission line.

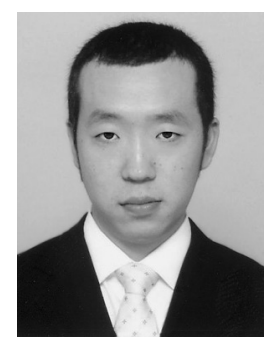

Tohlu Matsushima was born in Hyogo, Japan, on May 19, 1982. He received his M.E. degrees in Communication Network Engineering from Okayama University, Okayama Japan, in 2006. He is currently doctoral student of Okayama University. His research interest is electromagnetic interference problem. He is a student member of the Japan Institute of Electronics Packaging. 


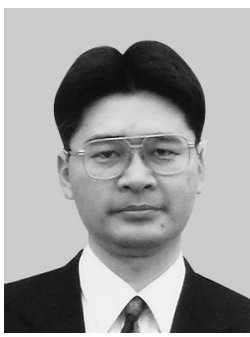

Tetsushi Watanabe was born in Okayama, Japan, on May 11, 1967. He received his B.E., M.E. and Ph.D. degrees in Electronics Engineering from Okayama University, Japan, in 1990, 1992 and 2004, respectively. Since 1992, he has been with the Industrial Technology Center of Okayama Prefecture. He has engaged in a study of immunity on LAN cables, measurement systems of EMC, and EMI reduction techniques. $\mathrm{He}$ is a member of the Japan Institute of Electronics Packaging.

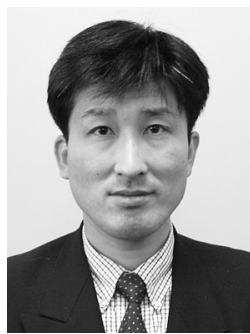

Yoshitaka Toyota was born in Okayama, Japan, on September 17, 1968. He received his $\mathrm{Ph} . D$. degree in electronic engineering from Kyoto University, Japan, in 1996. From 1996 to 1998, he was with Yokogawa Electric Co., Ltd. and in 2005 he worked at Georgia Tech as an overseas research scholar of the Ministry of Education, Culture, Sports, Science and Technology (MEXT) of Japan. He is currently an Associate professor at Okayama University. His research interest includes EMC design in highspeed digital systems. He is a member of IEEE, Japan Institute of Electronics Packaging, and the Japan Society of Applied Physics.

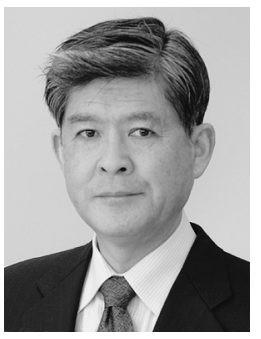

Ryuji Koga was born in Tokyo on $1945 . \mathrm{He}$ got the Doctorate from Kyoto University, and in 1976 he was with Okayama University, starting studies on electronics. On the way of developing a laser-sensing system, he was suffered from EMI from digital system attached it. He has then been engaged in the EMC problem. $\mathrm{He}$ is now Professor of Okayama University, and has experienced the chairperson of the Technical Committee of EMC, The Institute of Electronics, Information and Communication Engineers, JAPAN. Now he is the chair of IEEE EMCS Japan chapter. He will also chair the coming symposium EMC'09/Kyoto.

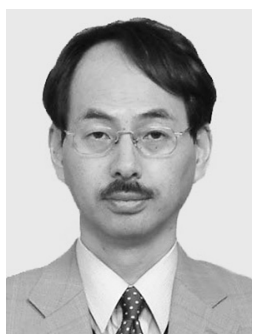

Osami Wada was born in 1957. He received the B.E., M.E. and Dr.E. degrees in electronics from Kyoto University, Japan, in 1981 1983 and 1987, respectively. Between 1988 and 2005 he was working at the Faculty of Engineering of Okayama University, Japan. Since 2005 he is Full Professor at the Department of Electrical Engineering at the Graduate School of Engineering of Kyoto University, Kyoto Daigaku Katsura, Nishikyoku, 615-8510 Japan. Prof. Wada is a member of IEE of Japan, the Japan Institute of Electronics Packaging, and IEEE. 